\title{
The Effect of Adinkra Symbol Patronage on Tourism Growth in Ghana: The Mediating Role of Youth Awareness
}

\author{
Zhu Zhe ${ }^{*}$ Solomon Bawuah \\ School of Art, Jiangsu University, Zhenjiang, 212013, China
}

\begin{abstract}
The study assessed the relationship between the patronage of adinkra symbols (AP) and the growth of Ghana's tourism sector (TG), through the mediating effect of youth awareness (YA), using descriptive statistics and multiples regression analysis and the PROCESS Micro analytical tool of IBM SPSS. Results showed that for every unit increase in youth awareness (YA), there is a $66.90 \%$ chance of increasing tourism growth (TG) in Ghana, provided that all other factors are held constant. Also, when tourists patronize more products with adinkra symbols, a unit increase in AP will cause about $81.40 \%$ chance of increasing the growth in tourism (TG) along the areas studied in Ghana. Further assessment of the mediating effect of YA, increased AP increases TG and the effect of AP on TG is higher when youth are more aware about adinkra symbols, thus when YA increased. It is recommended that more attention is focused on the relevance of youth education on the cultural heritages and adinkra symbols, and that the Ghanaian government invests more in value creation and marketing of the adinkrarelated tourism industry.
\end{abstract}

Keywords: Adinkra symbols, cultural heritage, youth awareness, tourism.

DOI: $10.7176 / \mathrm{JCSD} / 52-05$

Publication date:October $31^{\text {st }} 2019$

\section{Introduction}

According to the Oxford Business Group report in 2019, Ghana's tourism sector has significant potential to create jobs and drive economic development. This is so because the sector has been an area of focus for investment in Ghana (Oxford Business Group, 2019). The Bank of Ghana, in its reports in 2019, lists the tourism sector as fourthhighest earner of foreign currency following gold, cocoa and remittances (https://bog.gov.gh/statistics/publication/annual-report). In 2015, the tourism sector's foreign exchange earnings were estimated at USD 2.2 million with arrivals of tourists around 1.2 million, and contributed approximately $4.8 \%$ to Ghana's GDP. Concerning job creation, the sector employed about 393,000 Ghanaians in sectors such as hotels, travel trade, restaurants, entertainment, recreational, and tourist sites among others (Ekow, 2017).

The goal of the Ghana Tourism Authority is to "develop Ghana as an internationally competitive and highquality destination through partnerships, investments, aggressive marketing and conservation of the country's rich cultural, historical and environmental heritage". Stemming from the glimpses of hope for the tourism sector, it is important that the tourism sector takes advantage of all the resources available with touristic potential and harness their functionalities. Also, key challenges affecting the tourism sector such as poor hygiene and sanitation, pollution, poor hospitality services, low level of tourism awareness among youth of Ghana, poor road networks linking tourism sites to the rest of the country, low conservation of cultural heritage, inadequate infrastructure, adoption of foreign cultures and others. Among these aforementioned challenges is the issue of cultural heritage, which is the main focal point for this study.

Despite the many facets of tourism such as natural parks and reserves, rivers and waterfalls, mountains, beaches, hotels and restaurants, castles, and structures of colonial history among others, the fact remains that, most tourists complete their touristic experiences with symbolic items that are characteristic of visited destinations. These items connote the cultural heritage, details to the way a certain people live, and the ideologies they believe in. In Ghana, the Adinkra symbols are of rich cultural knowledge and meaning that are fascinating to foreigners, attracting many of them, and being a promising area for business creation and youth employment (AboagyewaaNtiri, et al., 2018; Kumekpor, 2002; Mato, 1986). These symbols carry conserved wisdom and knowledge that were transferred from many centuries ago, and which speak to the minds of those who understand it, often having positive psychological effects on those who pay attention to them (Adom, 2016; Wilburn, 2012; Willis, 1998; Rattray, 1927). They have the ability to confer aesthetic value as well as give information in serving as a means of communication (Adom et al., 2018). A study was conducted into the potential of well-known Adinkra symbols for use in heightening environmental sustainability awareness and education by way of an interactive communication (Moriarty, 2011).

The cultural heritage of the people of Ghana, their norms, and values as well as religious believes are embedded in these Adinkra symbols (Aboagyewaa-Ntiri, et al., 2018; Willis, 1998; Rattray, 1927). It can therefore be said that, a conscious effort at understanding the role played by Adinkra symbols on the growth of the tourism sector in Ghana, is a step towards improving the tourism sector from research point of view. This makes knowledge available on the effects of the Adinkra symbols and informs the level of seriousness that the sector needs for 
tourism growth consideration at a national level. The objective of this study is to specifically assess the level of awareness about Adinkra symbols among tourists and Ghanaian youth, the patronage of Adinkra items and how they influence growth in tourism.

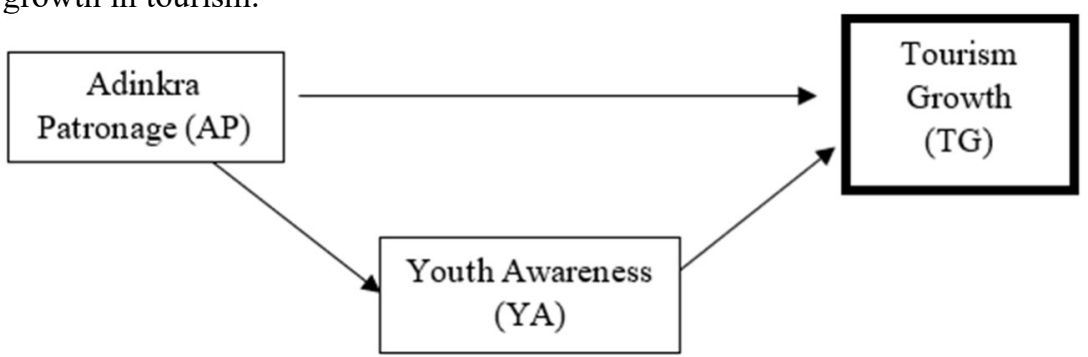

Figure 1: Conceptual framework depicting relationships between study variables

From the concept described (Figure 1), the study explores the relationship between the patronage of adinkra symbols (AP) and the growth in Ghana's tourism sector (TG). However, it also examines the effect of the level of awareness the youth have concerning the adinkra symbols on the relationship between AP and TG, referred to as, mediation. Thus, AP and YA are the predictor or independent variables whereas TG is the response or dependent variable in this study.

Table 1: Some Adinkra symbols and their meanings

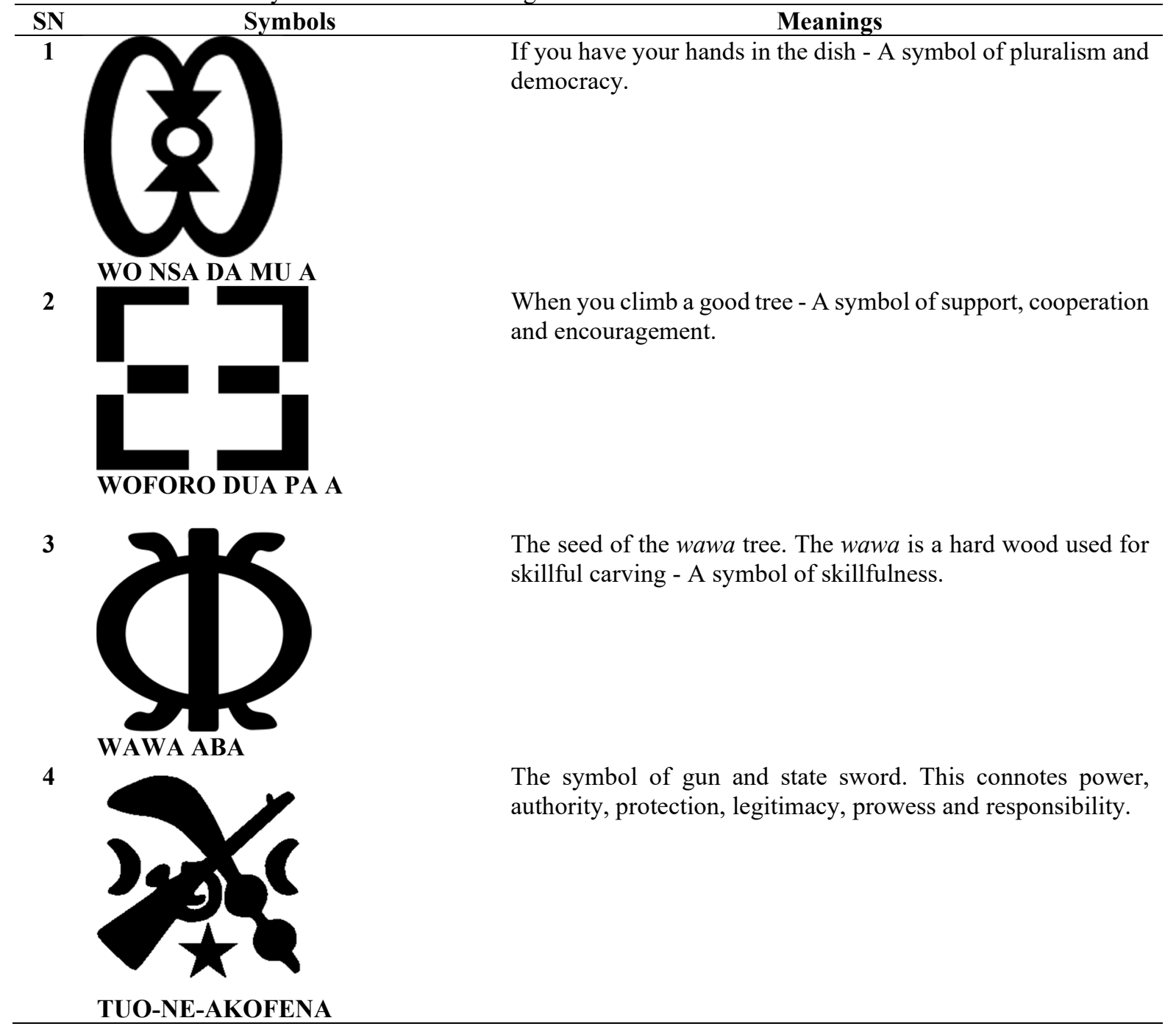




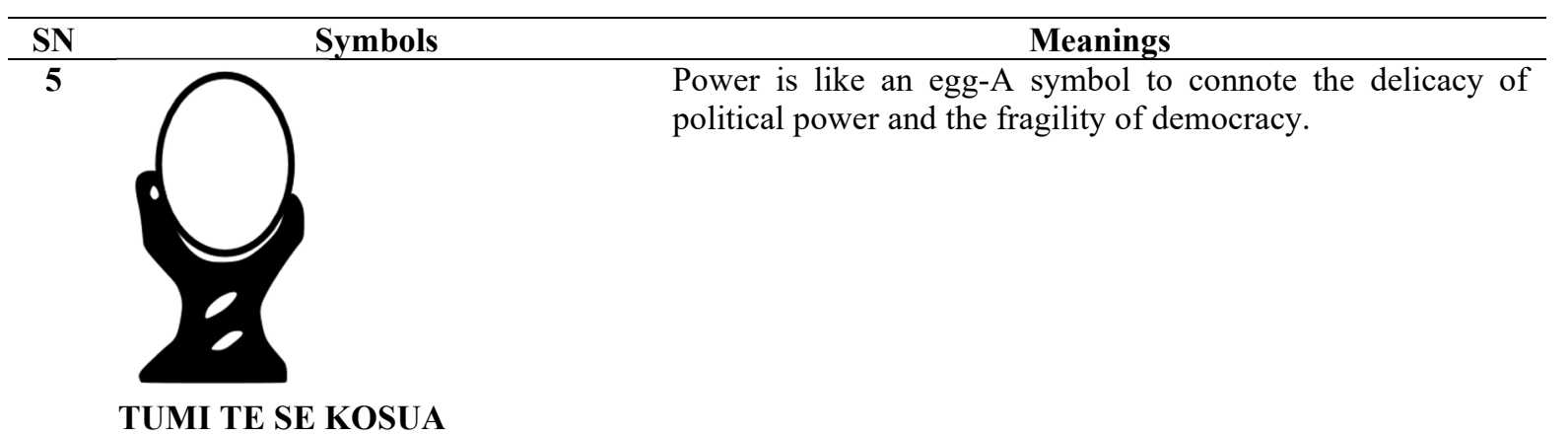

6

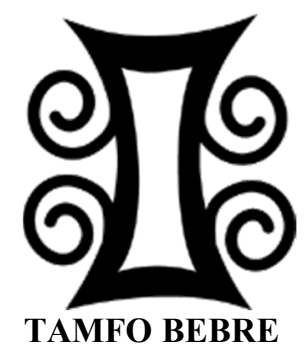

7

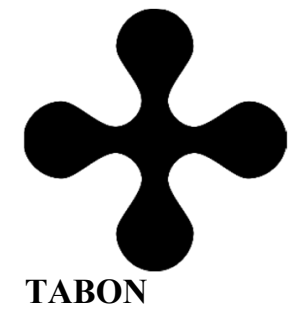

8

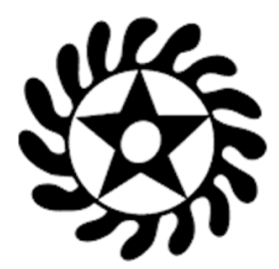

9
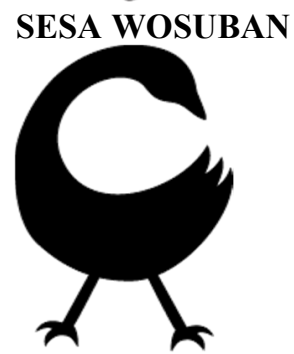

10

\section{SANKOFA}

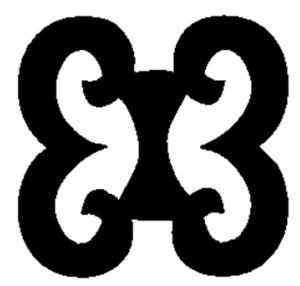

The enemy shall suffer-A symbol of jealousy.

A symbol of a paddle, depicting courage and hard work.

Change your lifestyle- A symbol of transformation and change.

Return and take it-A symbol depicting the need for learning from past to improve the future, correcting past mistakes and wisdom.

Preparedness- A symbol depicting readiness and unity.

\section{Methodology}

The study followed a quantitative, cross-sectional survey approach. Five-hundred (500) youth who sell different types of items bearing Adinkra symbols to tourists were randomly selected and involved in this study as respondents. The study location was purposively selected to be the Kumasi Centre for National Culture and Ntonso, 
all located in the capital city of Ashanti region of Ghana. Structured questionnaires were administered to respondents with a return rate of $94 \%$. Data were organized in IBM-SPSS (Version 25) and responses for all variables were measured using a 5-point Likert scale [(5) strongly Agree, (4) Agree, (3) neither Agree nor Disagree, (2) Disagree, (1) Strongly Disagree]. Mean values of responses and standard deviations were computed. Data were assessed using descriptive statistics and correlational analysis. The PROCESS macro function in IBM-SPSS as described by Hayes (2018) was used to establish the relationships between all variables AP, TG (Figure 1) and the mediating function of YA. Results are presented in Tables and Figures.

\section{Results and discussions}

3.1 Statistical diagnostics for data fitness

Distribution of data was normal as depicted in Figure 2 and fitness of data for regression was carried out using correlation matrix between variables to assess multiple collinearity (Table 1). Residual plots of expected versus observed data were also used to approve the appropriateness of the statistical approach adopted in this study (Figure 3 ). Mean values for variables were $28.06 \pm 2.11,31.22 \pm 1.13$ and $22.35 \pm 3.28$ for TG, AP and YA respectively.

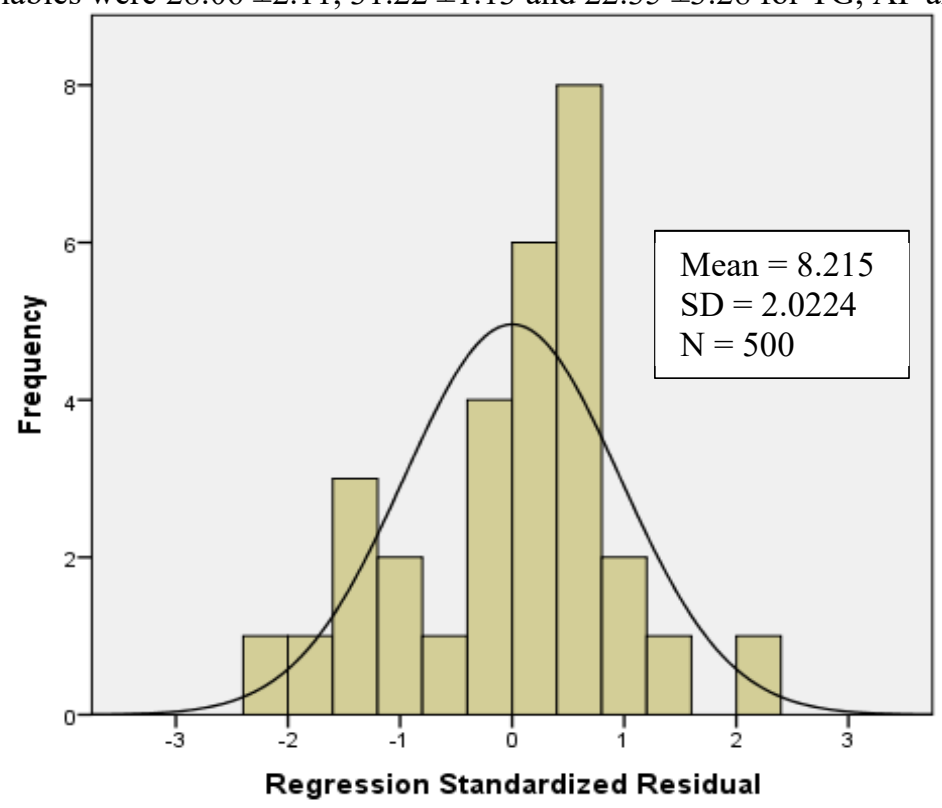

Figure 2: Distribution of data

The normal distribution of data confirmed the suitability of data for the multiple regression analysis.

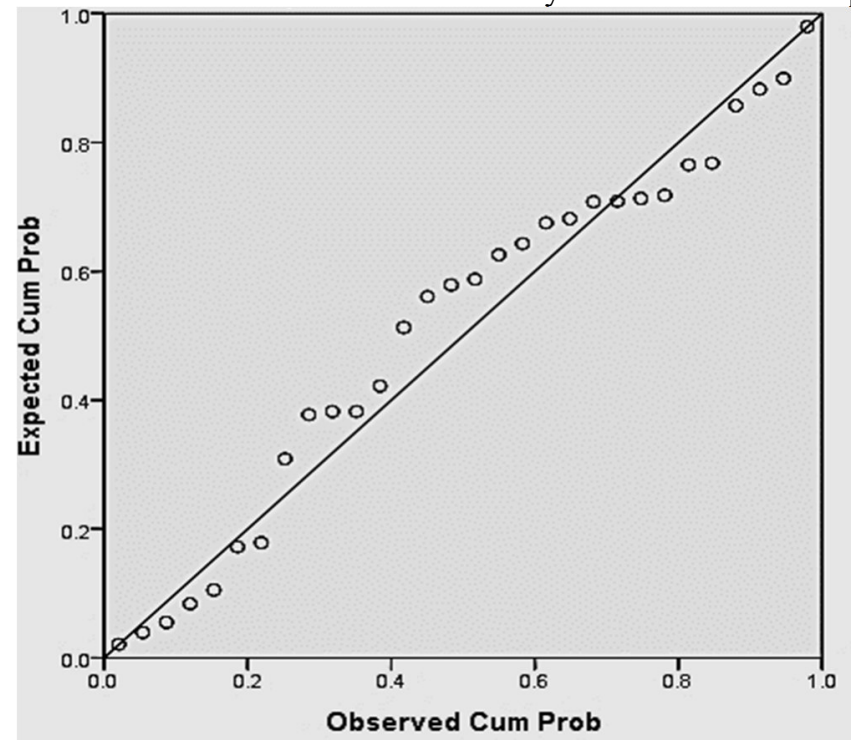

Figure 3: Regression residual plot of expected versus observed data

3.1.1 Multi-collinearity test between variables

Multi-collinearity test was performed using the correlation matrix of variables to assess the relationships between all variables. The relationships between the two independent variables (YA and AP) and the dependent variable 
(TG) were significant $(\mathrm{P}<0.05)$, showing that the regression model was fit (Table 2$)$.

Table 2: Correlation matrix

\begin{tabular}{llrrr}
\hline & & TG & AP & \multicolumn{1}{c}{ YA } \\
\hline TG & Pearson Correlation & 1 & $.814^{* *}$ & $.669^{* *}$ \\
& Sig. (2-tailed) & & .000 & .000 \\
& N & 500 & 500 & 500 \\
AP & Pearson Correlation & $.814^{* *}$ & 1 & .202 \\
& Sig. (2-tailed) & .000 & & .039 \\
& $\mathrm{~N}$ & 500 & 500 & 500 \\
YA & Pearson Correlation & $.669^{* *}$ & .202 & 1 \\
& Sig. (2-tailed) & .000 & .039 & 500 \\
& $\mathrm{~N}$ & 500 & 500 & \\
$*$ **. Correlation is significant at the 0.05 level (2-tailed). & & \\
\hline
\end{tabular}

\subsection{Regression analysis of data}

The regression model was significant $(\mathrm{P}<0.05)$ and meets the demands of this study, and fit for establishing the relationships between dependent and independent study variables. Model summary information and analysis of variance (ANOVA) confirmed the statistical significance with $\mathrm{R}^{2}$ and adjusted $\mathrm{R}^{2}$ values of 0.721 and 0.699 respectively. This further proves that the model was adequate as the independent variables were able to predict the variability measured in the dependent variables by $72.10 \%$ (Table 3 ).

Table 3: Model Summary ${ }^{\mathrm{b}}$

\begin{tabular}{lcrrr}
\hline Model & R & R Square & Adjusted R Square & Std. Error of the Estimate \\
\hline 1 & $.816^{\text {a }}$ & $\mathbf{. 7 2 1}$ & $\mathbf{. 6 9 9}$ & 2.23521 \\
a. Predictors: (Constant), AP, YA & & \\
b. Response Variable: TG & & \\
\hline
\end{tabular}

Table 4: Model ANOVA ${ }^{\mathrm{a}}$

\begin{tabular}{|c|c|c|c|c|c|c|}
\hline \multicolumn{2}{|c|}{ Model } & Sum of Squares & $\mathrm{df}$ & Mean Square & $\mathrm{F}$ & Sig. \\
\hline 1 & Regression & 168.226 & 2 & 61.738 & 27.118 & $.003^{b}$ \\
\hline & Residual & 80.092 & 496 & 2.665 & & \\
\hline & Total & 248.318 & 498 & & & \\
\hline & $\begin{array}{l}\text { sponse Vari } \\
\text { edictors: (C }\end{array}$ & $\begin{array}{l}\mathrm{TG} \\
\text { ant) AP YA }\end{array}$ & & & & \\
\hline
\end{tabular}

Table 5: Model Coefficients ${ }^{\mathrm{a}}$

\begin{tabular}{|c|c|c|c|c|c|c|}
\hline \multirow{2}{*}{\multicolumn{2}{|c|}{ Model }} & \multicolumn{2}{|c|}{ Unstandardized Coefficients } & \multirow{2}{*}{$\frac{\text { Standardized Coefficients }}{\text { Beta }}$} & \multirow[t]{2}{*}{$\mathrm{t}$} & \multirow[t]{2}{*}{ Sig. } \\
\hline & & $\mathrm{B}$ & Std. Error & & & \\
\hline 1 & (Constant) & 7.714 & 3.821 & & 2.030 & .010 \\
\hline & YA & .669 & .134 & .669 & 3.109 & .004 \\
\hline & AP & .814 & .082 & .814 & 4.001 & .009 \\
\hline
\end{tabular}

a. Dependent Variable: TG

The general regression equation, $\hat{\mathbf{Y}}=\boldsymbol{\beta}_{\mathbf{0}}+\boldsymbol{\beta}_{1} \mathbf{X}_{\mathbf{1}}+\boldsymbol{\beta}_{2} \mathbf{X}_{2}+\boldsymbol{E}$ was used for the analysis with beta values representing the coefficients of the independent variables and the constant whereas the $\hat{\mathbf{Y}}$ term represents the observed outcome of the model. Assuming a zero error, the predicted equation becomes $\mathbf{T G}=\boldsymbol{\beta}_{\mathbf{0}}+\boldsymbol{\beta}_{\mathbf{1}} \mathbf{Y} \mathbf{A}+\boldsymbol{\beta}_{\mathbf{2}} \mathbf{A} \mathbf{P}$, where $\beta_{0}, \beta_{1}$ and $\beta_{2}$ are the coefficients for the constant, YA and AP respectively. The coefficient terms are available in Table 5, and substituting their values into the predicted equation, it becomes $\mathbf{T G}=\mathbf{7 . 7 1 4} \pm \mathbf{3 . 8 2 1}+\mathbf{0 . 6 6 9} \pm \mathbf{0 . 1 3 4 Y A}+$ $\mathbf{0 . 8 1 4} \pm \mathbf{0 . 0 8 2 A P}$. From this predicted model equation, it can be said that for every unit increase in youth awareness (YA), there is a $66.90 \%$ chance of increasing tourism growth (TG) in Ghana, provided that all other factors are held constant. Also, when tourists patronize more products with adinkra symbols, a unit increase in AP will cause about $81.40 \%$ chance of increasing the growth in tourism (TG) along the areas studied in Ghana, when all other factors are not interacting.

\subsection{Mediation assessment}

Using the PROCESS macro application of Hayes (2018) in SPSS, results show a good correlation between YA and TG with a significant correlation coefficient of $0.599(\mathrm{P}<0.05)$. 


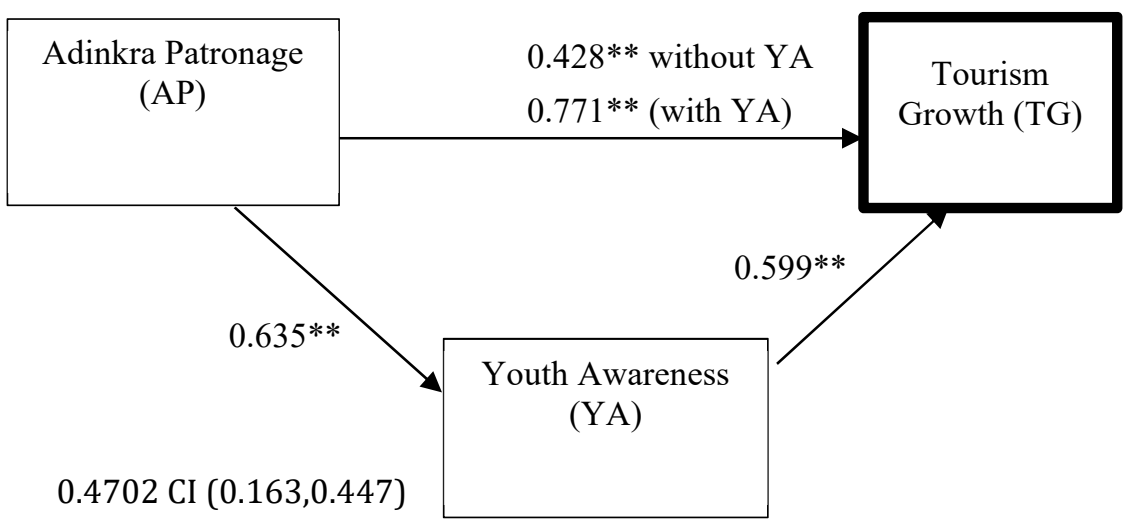

Figure 4: Mediation role of YA on the relationship between AP and TG.

From Figure 4, mediation test confirms a strong relationship between YA, TG, and shows that the relationship between AP and TG is more significant when YA mediates (0.771) rather than in its absence (0.428) (Hayes, 2018). Also, the indirect effect of AP on TG through the mediating role of YA was 0.4702 , which was higher than the direct effect of AP on TG (0.428) (Figure 4). This means that, although the patronage of Adinkra symbols (YA) has a significant effect on tourism growth (TG) in Ghana, it does so more when the youth are more aware of Adinkra symbols. The objective of the cultural policy of Ghana is "to enhance Ghanaian cultural life and develop cultural programmes to contribute to the nation's human development and material progress through heritage preservation, conservation, promotion and the use of traditional modern arts and crafts to create wealth and alleviate poverty" (Ghana cultural policy, 2004). The keywords that are relevant to this study are "heritage preservation, conservation, promotion......traditional modern arts and crafts...".

According to reports from the United Nations (UN) (2013), the culture of a society has the power to transform it entirely, strengthen local communities and nurture a sense of identity and citizenship for people of all ages. Culture was seen as a vector for youth development and civic engagement in the promotion of sustainable social and economic development. The role of youth in acting as a bridge between cultures and being peace-promoting agents for intercultural understanding is partly highlighted by their keen understanding of adinkra symbols that represent the cultural heritage of Ghana. The relevance of this linkage to the growth of the tourism sector of Ghana, and in the generation of employment cannot be overemphasized. From this study, results have highlighted the relevance of youth awareness about adinkra symbols to the development of tourism in Ghana.

The UNESCO World Heritage Education Program, started in 1998, recognizes the crucial role of young people in community development. The program gave youth the opportunity to acquire knowledge about protection, conservation and promotion of culture, encouraging them by building their capacities to become thinkers and actors of national and communal development (http://whc.unesco.org/en/educationkit/). A recent study was conducted to "investigate into the possibility of using the philosophical ideologies enshrined in the Adinkra symbols for educating the Ghanaian people about environmental sustainability" (Dickson Adom, et al 2018). Works of the African Union, the United Nations, UNESCO, Ghanaian government and other international organizations have emphasized the need to empower the youth of Africa and Ghana for that matter, for sustainable development (African Union, 2011; European Commission, 2011; Ministry of Youth and Sports, Ghana, 2010; UNESCO, 2010). This is one major aspect of reduction in youth unemployment in the sub region (National Treasury, Ghana, 2010) and this is in line with the cultural policy of Ghana, to create jobs and enhance the conservation, protection and use of modern traditional arts and crafts. Such objectives have also been added to the education curricula of schools in Ghana to encourage awareness creation among the youth. These efforts buttress the fact that studies of this nature are not only in the interest of satisfying research but also in the interest of relevant issues of socio-economic concern to Ghana and Africa as a whole, and by extension, to other parts of the world regarding cultural heritage and youth empowerment and development (Tagoe and Oheneba-Sakyi,2015; UN, 2005).

\section{Conclusion}

The study assessed the relationship between the patronage of adinkra symbols (AP) and the growth of Ghana's tourism sector (TG), through the mediating effect of youth awareness (YA), using descriptive statistics and multiples regression analysis and the PROCESS Micro analytical tool of IBM SPSS. Results from multiple regression showed that for every unit increase in youth awareness (YA), there is a $66.90 \%$ chance of increasing tourism growth (TG) in Ghana, provided that all other factors are held constant. Also, when tourists patronize more products with adinkra symbols, a unit increase in AP will cause about $81.40 \%$ chance of increasing the growth in 
tourism (TG) along the areas studied in Ghana, when all other factors are not interacting. Further assessment of the mediating effect of YA, increased AP increases TG and the effect of AP on TG is higher when youth are more aware about adinkra symbols.

It is recommended that government programs and policies target investment in cultural heritage enhancement, promotion and education, specifically, the use of adinkra symbols and their meanings to the Ghanaian people. Further research is needed to understand the effect of these symbols on tourists' perception of Ghana and on their individual lives.

\section{ACKNOWLEDGEMENT}

The researchers thank sincerely, the traditional elders and workers of Kumasi Centre for National Culture and Ntonso all in the Ashanti region of Ghana for their maximum contribution towards the research.

\section{References}

1. Aboagyewaa-Ntiri, J., Campion, C.B., \& Kemevor, A.K. (2018). Extension of knowledge on Ghanaian adinkra symbols in relation to Maslow's theory. International Journal of Network and Communication Research, 5(1), 11-24.

2. Adom, D., Asante, E.A., \& Kquofi, S. (2016). Adinkra: an epitome of Asante philosophy and history. Research on Humanities and Social Sciences, 6(14).

3. Adom, D., Opoku, M., Newton, J.P., Yeboah, A. (2018). Adinkra Cultural Symbols for Environmental Sustainability Education in Ghana. World Environment, 8(2): 36-46. https://doi.org/10.5923/j.env.20180802.02.

4. Bank of Ghana Report (2019). Available at: https://bog.gov.gh/statistics/publication/annual-report. Accessed 17/04/2019 at 12:04.

5. Ekow, S. (2017). Ghana's tourism potential. Available at: https://www.icao.int/Meetings/SUSDEVAT/Documents/Presentation GHANA\%20TOURISM\%20POTENTIALS.pdf. Accessed 13/04/2019 at 18:29.

6. Kumekpor, K.B. (2002). Research Methods \& Techniques of Social Research, SonLife Printing Press and Services, Ghana.

7. Mato, D. (1986). Clothed in symbol-The art of Adinkra among the Akan of Ghana. Indiana: Bloomington.

8. Moriarty, J. (2011). Qualitative methods overview (SSCR methods reviews). National Institute for Health Research School for Social Care, London.

9. Oxford Business Group Report (2019). Ghana's tourism sector. Available at: https://oxfordbusinessgroup.com/ghana-2019/tourism. Accessed 13/04/2019 at 18:27.

10. Rattray, R. S. (1927). Religion and art in Ashanti. London: Oxford University Press.

11. Wilburn, K. (2012). Africa to the world! Nkrumah-era philatelic images of emerging Ghana and PanAfricanism, 1957-1966. African Studies Quarterly, 13, 23-32.

12. Willis, B. W. (1998). The Adinkra dictionary: A visual primer on the language of Adinkra. Washington DC: The Pyramid Complex.

13. World Heritage in Young Hands. Available at: http://whc.unesco.org/en/educationkit/. Accessed 17/04/2019 at 11:49.

14. Tagoe, M.A., \& Oheneba-Sakyi, Y. (2015). Harnessing the power of the youth through national youth policies in Ghana: challenges to notions of empowerment. Contemporary Journal of African Studies, 3(1), 69-91.

15. United Nations (2005). World Youth Report: Young People Today, and in 2015. Department of Economic and Social Affairs [DESA]. New York, USA.

16. UNESCO (2010). UNESCO SHS Strategy on African Youth: Towards an Enabling Policy Environment for Youth Development and Civic Engagement in Africa (2009-2013). Executive Board, 184 Session. Paris, France.

17. National Treasury. (2011). Confronting Youth Unemployment: Policy Options for South Africa. Discussion Paper, February, Pretoria, South Africa.

18. Ministry of Youth and Sports (2010). National Youth Policy of Ghana: Towards an Empowered Youth, Impacting Positively on National Development. August. Accra: Ghana.

19. Economic Commission for Africa (2011). African Youth Report: Addressing the Youth Education and Employment nexus in the New Global Economy. United Nations Economic Commission for Africa. Addis Ababa: Ethiopia.

20. African Union (2011). African Youth Decade 2009-2018 Plan of Action: Accelerating youth empowerment for sustainable development. Addis Ababa, May 2011.

21. Ghana Cultural Policy. Available (2004). http://www.ghanaiandiaspora.com/wp/wpcontent/uploads/2014/09/CULTURAL.-POLICY-FINAL.pdf. Accessed 19/04/2019 at 10:44. 